\title{
ERITROBLASTOSIS FETAL (Factor Rh)
}

\author{
Dr. ERnesto Plata Rueda
}

El presente trabajo se refiere a las anotaciones recogidas sobre casos de eritroblastosis fetal (anemia hemolitica) ocasionada por isoinmunización anti Rh. Corresponden a la Clínica "David Restrepo", Instituto Materno Infantil y clientela civil.

Se trata de una actualización sobre el tema que deja de lado las consideraciones generales por estimar que son ya del dominio de todos los obstetras y pediatras modernos.

El trabajo se subdivide en anotaciones sobre antecedentes, embarazo, parto, investigación y diagnóstico de la enfermedad, tratamiento y resultados.

Las observaciones sobre isoinmunización anti A y técnica de la exanguinotransfusión serán objeto de futuras publicaciones.

Antecedentes.-La multiparidad sigue siendo un dato de especial interés para el diagnóstico de eritroblastosis por factor $\mathrm{Rh}$. De nuestros casos, todos han sido de tercer hijo en adelante, con una mayor incidencia para el cuarto hijo y un caso considerado ya raro en el noveno parto. Sin embargo, al estudiar los antecedentes, encontramos eritroblastosis en el segundo hijo en cinco de las pacientes.

Muchos obstetras desechan la investigación del factor $\mathrm{Rh}$ en la primigestante por la escasísima incidencia de eritroblastosis $\epsilon n$ el primer hijo. Tal negligencia no se justifica de ninguna manera si se tiene en cuenta que se han publicado casos espontáneos de isoinmunización en el primer embarazo y además, no siempre es fácil obtener el dato de transfusiones previas al embarazo, sobre todo en la niñez, las cuales han podido ser incompatibles. En los antecedentes de nuestro caso 3 encontramos el dato muy curioso por cierto de eritroblastosis en el segundo hijo, ocasionada porque a la madre le aplicaron durante el primer embarazo, sangre del esposo por vía intramuscular para el trata- 
miento de hiperemesis gravidica (?). Otros dejan de lado la investigación en la gran muitípara por considerar que "ya no pasara nada"; sin embargo, tenemos un ejemplo de eritroblastosis en el noveno parto (caso 9). Cabe aquí destacar la importancia que tuvo para esta madre el hecho de que su octavo embarazo terminó por aborto tratado con legrado uterino; posiblemente esta maniobra "sembró" glóbulos rojos Rh positivos en la circulación uterina, determinando la isoinmunización que afectó al noveno hijo.

Puntualizado así io que se refiere al número de la gestación, veamos ahora lo que se refiere a algunos detalies de mucho interés en las gestaciones previas. Como lo veremos luego más detenidamente, es de importancia fundamental para definir la conciucta durante el embarazo, en el parto y con el niño, la determiración de si existen antecedentes de mortinatos o muertes perinatales sospechosas o confirmadas de ser producidas por eritroblastosis. En 8 de nuestras pacientes existía tal antecedente y ello permitió la ejecución de la exanguinotransfusión en las primeras horas, una vez obtenidos los primeros datos de laboratorio, con notable mejor a en los resultados. Parece definitivamente demostrado que por lo menos dentro del mismo Rh, el aborto habitual no es producido por la inmunización (1).

De nuestras 15 pacientes, 9 tienen antecedentes indudables de hijos eritroblastósicos y la investigación se imponía; en otras tres fue la evidencia clínica la que determinó la investigación y solamente en tres, gracias al control minucioso llevado por el obstetra, permitió descubrir la enfermedad en sus comienzos, sin que existieran antecedentes. Estas cifras ponen de manifiesto lo muy poco que se piensa todavía en nuestro medio en la Eritroblastosis, pese a que es de todos conocida la importancia que tiene su diagnóstico precoz.

En nuestra primera publicación acerca de eritroblastosis (2), hablamos de la relativa baja incidencia de mujeres $\mathrm{Rh}$ negativas en la clientela obstétrica. Ahora podemos hacer esta otra observación; en la clientela civil de la clase acomodada, la frecuencia es elevada, aunque no tanto como en los Estados Unidos por ejemplo $(15 \%)$. En la clase media económica la incidencia oscila entre 6 y $7 \%$ (Clínica David Restrepo por ejemplo). En la clientela de hospital de caridad (Instituto Materno Infantii), apesar de que el factor Rh no se investiga sistemáticamente (como debería hacerse), el hecho de que a pesar de estar permanentemente sobre la investigación de las ictericias, no haya dado lu- 
gar sino al descubrimiento de unos pocos casos sobre un volumen enorme de nacimientos, permite suponer que la incidencia de madres $\mathrm{Rh}$ negativas sea realmente escasa, posibiemente por lactor racial (mayor proporción de individuos con sangre indigena?).

Consideraciones sobre el embarazo.-Actualmente se considera un axioma de la Obstetricia que toda gestante debe ser investigada para el factor $R h$. Teniendo en cuenta que es el tipo Rho el más inmunizante, siéndolo mucho menos los tipos Rhl y $\Omega h 2$, en algunos hospitales, por motivos económicos y de tiempo, se vienen omitiendo el uso de los sueros Anti-rh' y Anti-rh". Pese a la escasa frecuencia de eritroblastosis causada por estos factores (nosotros no tenemos ninguna), siempre es interesante una clasificación lo más completa posible. En efecto, si bien es cierto que al resultar la gestante negativa con el suero Anti-Rho, esto es motivo suficiente para someterla a toda la investigación para la eritroblastosis, no por ser positiva con este suero solamente puede omitirse dicha investigación, ya que puede ser negativa con el Anti-rh' o el Anti-rh" o ambos a la vez, con lo cual, si su esposo es positivo para algunos de estos últimos, existen posibilidades, aunque remotas, de eritroblastosis. En resumen, el resultado "Rho positivo" no es índice de seguridad para descartar a una gestante como productora de fetos eritroblastósicos.

Si la gestante resultare negativa para alguno de los tres sueros, el esposo debe someterse a una investigación análoga. Al efecto podemos hacer consideraciones semejantes: que el esposo de una gestante $\mathrm{Rh}$ (negativa) resulte también negativo con el suero Anti-Rho solamențe, no puede dar ninguna seguridad sobre que no vaya a presentarse eritroblastosis en ese matrimonio, porque puede ser positivo con los sueros Anti-rh' o Anti-Rh", para los cuales su esposa es negativa.

Aunque estas consideraciones parezcan triviales a quienes se encuentran muy familiarizados con estos asuntos, estamos seguros de que no son del dominio de todos los pediatras u obstetras, por lo cual no hemos dudado en consignarlas aquí.

Ei hallazgo de cualquier incompatibilidad $\mathrm{Rh}$ impone durante el embarazo el control de los anticuerpos en el suero materno. Ultimamente existe la tendencia a desechar la investigación de las aglutininas bivalentes o salinas por considerar que no representan peligro para el feto al no poder atravesar la barrera placentaria debido a su gran molécula. Sin embargo, por lo menos 
teóricamente, condiciones especiales de la placenta en un caso dado podrian permitir su paso al feto y perjudicarle, a más de que su hallazgo habla en favor de la capacidad de la madre para producir anticuerpos. De todos modos la investigación más importante es la de los anticuerpos monovalentes (glutininas o aglutininas albuminoideas). El método más usado hoy es el de la antiglobulina (Coombs Indirecto), por ser el más exacto. Sin embargo, como algunos laboratorios usan todavía otros métodos, debe exigirse la especificación de cual se usó, pues la sensibilicad de cada uno varia. Una unidad en el "Rh blocking test" equivale a 20 unidades en el " $\mathrm{Rh}$ conglutination Test", a 50 unidades en el "Antiglobulin test" (Coombs indirecto) y a 100 unidades en el "Proteolitic enzyme test". La falta de cuidado en el lavado de las pipetas puede ser la causa de títulos extraordinariamente elevados. Wiener nunca ha encontrado títulos de anti $\mathrm{Rh}$ superiores a 50.000 U. La sangre del cordón de nuestro caso 5 fue reportada como "intitulable" para el Coombs indirecto: creemos que fuera debido a un mal uso de las pipetas. El título más alto que hemos encontrado por el método de Coombs es el del caso 10 ( 1 x 128). Ultimamente se ha abreviado la designación de los títulos, mencionando en unidades la última dilución que da aglutinación (en el caso anterior, 128 unidades).

Desde un punto de vista práctico, la investigación de los anticuerpos puede hacerse solo al principio del embarazo, y al 8 o mes. Para trabajos de investigación, en instituciones de buenas disponibilidades, así como en casos especiales (fetos muertos in útero por ejemplo), la determinación dé los anticuerpos debe hacerse al principio del embarazo trimestralmente, y quincenalmente en las últimas 6 semanas; también en la sangre del cordón y post parto.

Sobre el tratamiento médico durante el embarazo no existe unanimidad de criterio y diversos procedimientos han sido recomendados. El ideal sería poder impedir que glóbulos rojos del niño pasaran a la madre a través de la placenta (cosa que ocurre normalmente en uno de cada tres embarazos normales), para jo cual por lo menos teóricamente el procedimiento de elección sería aquel que lograra una placenta lo más normal posible y relaciones normales utero-placentarias. Por esto se ha preconizado el uso de la progesterona y de la vitamina E. Ante el fracaso de esta tentativa se ha pensado impedir la producción de anticuerpos por medio de la cortisona y el ACTH o bien "hacerle competen- 
AMEIIA HBMOLITICA - PACTOR Rh.

\begin{tabular}{|c|c|c|c|c|c|c|c|c|c|c|c|c|c|c|c|}
\hline CASO & I & II & III & IV & $\nabla$ & VI & VII & VIIH & IX. & $\pi$ & $\overline{X I}$ & $\mathrm{XII}$ & $x I I I$ & IIV & $\pi V$ \\
\hline PARIDAD & IV & $\nabla$ & $\nabla$ & III & IV & VII & III & VII & $\mathrm{Ix}$ & IV & IV & IV & VII & III & IV \\
\hline ERITROBLASTOSIS DESDB & III & III & II & & III? & II & II & II & & III & III & II? & TV & & II \\
\hline MORTINATOS & - & - & - & - & - & 3 & - & - & - & - & - & - & - & - & - \\
\hline ABOBCTOS & - & - & 1 & - & 1 & - & - & - & 1 & - & - & - & 1 & - & - \\
\hline COONBS IIDIRECTO & $+\circ$ & 16 & 8 & - & + & 32 & 8 & 8 & 1280 & 123 & 4 & $16^{\circ}$ & 16 & 8 & 16 \\
\hline CONTROL EN EMBARAZO & - & + & + & + & + & + & + & * & - & + & 4 & - & + & + & + \\
\hline PAR T $0^{1}$ & $\mathbf{E P}$ & IT & BP & B & B & $\mathrm{CP}$ & $\mathrm{CP}$ & BP & $\mathrm{ET}$ & IP & ET & BT & BT & IT & IT \\
\hline ATBNCION FSOIATRICA & $3 d$. & Nao & Neo & $2 \mathrm{~d}$ & Nao & $\mathrm{Nac}$ & 820 & Nao & Nao & $8 d$ & $12 \mathrm{~d}$ & $3 d$ & Nao & Nac & Nac \\
\hline BILIRRDBINA IND. & & & & & & & $25^{\circ}$ & 20 & 13 & 4 & & 33 & & & \\
\hline$A N B$ I I A & + & & + & - & + & & & - & + & + & & - & + & & \\
\hline COOLBS DIRECTO & & + & + & & + & + & + & + & + & + & + & + & & + & + \\
\hline PRELATURO & + & - & + & - & - & + & - & + & - & - & - & - & & - & - \\
\hline ICTERICIA PRECOZ & + & & & $\bullet$ & • & & $+\cdot$ & - & + & + & + & + & & $+\cdot$ & $++^{\circ}$ \\
\hline ESPLENOMEGALIA & - & + & $+t+$ & + & + & + & - & - & + & + & + & + & + & - & - \\
\hline HEPATOMBGALIA & + & + & +++ & - & - & + & - & - & + & - & + & + & + & + & - \\
\hline EDE A $\mathrm{S}$ & - & + & + & - & - & - & - & - & - & - & + & - & +++ & - & + \\
\hline KERNICTERUS & + & $+\cdot$ & - & + & - & - & $+\circ$ & - & $+\bullet$ & - & - & + & & - & - \\
\hline ESTADO GENERAL & M & B & $\mathbf{u}$ & $\mathbf{R}$ & B & B & B & B & $\mathbf{R}$ & B & B & n & $y$ & B & B \\
\hline TRATADO A.... & $4 d$ & $\mathrm{Nac}$ & HaO & $6 d$ & $16 \mathrm{~h}$ & Nao & $\mathrm{NaO}$ & $24 \mathrm{~h}$ & 25h & $11 \mathrm{~h}$ & $24 \mathrm{~h}$ & $3 d$ & No & NaO & $4 \mathrm{~h}$ \\
\hline EXANGUINOTRANSFUSION & $\mathbf{S R}$ & 0 & $\delta$ & $\mathbf{S R}$ & $\mathrm{U}$ & U & U & U & 0 & 0 & 0 & U & & U & $\mathrm{U}$ \\
\hline RESULLADOS ${ }^{3}$ & $\mathbf{R}$ & $\mathbf{R}$ & $\underline{y}$ & $\mathbf{R}$ & B & $\mathbf{R}$ & B & B & B & B & B & $M$ & y & B & B \\
\hline
\end{tabular}

I - Bz Espontánoo - $I_{2}$ Inducido - $T_{2}$ a Tórmino - $P_{2}$ Prematuro - $C_{2}$ Cesárea.

2 - Ut VIa Umbilical - SR: U6todó Safona-Radial.

3 - Bt Bueno - Mt Muerto - Ri Rotardo nourologico discroto.

- Post transfusion O Post parto.

cia" al antígeno $\mathrm{Rh}$ por medio de otros antígenos bacterianos (vacuna tífica, pertussis). El hapteno Rh (porción no antigéni(a del aglutinógeno $\mathrm{Rh}$ ), inyectado a la madre durante el embarazo, haría que se fijaran sobre él los anticuerpos $\mathrm{Rh}$ protegiendo así el feto; pero los resultados clínicos han sido desalentadores. En nuestras pacientes de la Clínica David Restrepo se ha usado en forma rutinaria la administración de hormona del cuerpo luteo por las razones expuestas antes, en asociación con vitamina $\mathrm{K}$ de cuya eficacia se ha hablado. La titulación seriada de los anticuerpos en el curso del embarazo no permite de ninguna manera apreciar sus efectos, pues los títulos de éstos son de por sí muy variables. Los casos 3,6 y 8 impresionan un poco en 
cuanto a los resultados, especialmente el número 6, una madre que había perdido 5 hijos, tres de ellos muertos in útero, a quien se logró llevar la gestación hasta los $71 / 2$ meses con feto vivo que fue salvado gracias a ia exanguinotransfusión (Las muertes intrauterinas habían ocurrido alrededor del $7^{\circ}$ mes). La aplicación de vacuna pertussis y tífica está indicada en las gestantes $\mathrm{Rh}$ negativas no isoinmunizadas.

Consideraciones sobre el parto.-Existe también controversia sobre la conveniencia o inconveniencia de inducir el parto prematuro en las gestantes $\mathrm{Rh}$ negativas isoinmunizadas. Después de que se abusó del intervencionismo provocando partos prematuros inclusive en pacientes sin pruebas de isoinmunización, se ha caído ahora en el extremo opuesto tendiente a dejar llegar al término todas las gestaciones. No nos parece juiciosa tal conducta extremista y consideramos que, si existen antecedentes de hijos eritroblastósicos y con mayor razón de muertes intrauterinas por esta causa, está indicado provocar el parto prematuro, procurando llevar al feto al máximo de viabilidad, es decir, no antes de $\operatorname{los} 7 \frac{1}{2}$ meses. En cuatro de nuestras pacientes se ha provocado el parto prematuro con resultados evidentemente buenos. En el caso 6, ya comentado, el antecedente de numerosos mortinatos requería un parto lo antes posible; la paciente fue hospitalizada desde los $61 / 2$ meses y sometida a una dieta hiper calórica con aplicación de soluciones dextrosadas por vía venosa, con el ánimo de propender el aumento de tamaño del feto; fue así como a los $7 \frac{1}{2}$ meses se obtuvo una niña de 2.260 gramos que toleró perfectamente la exanguinotransfusión. Los enemigos de esta conducta alegan que la eritroblastosis tiene mayor tendencia a producir kernicterus en el prematuro que en al niño a término; si bien esto es verdad, nos ha parecido una conducta más dinámica en esos casos especiales, provocar el parto prematuro y practicar una exanguinotransfusión oportuna, que esperar pasivamente a que el niño nazca muerto. Nadie puede negar que entre más tiempo permanezca el niño dentro del útero materno, más expuesto estará a la acción letal de los anticuerpos; todos hemos visto por otra parte cómo suben los títulos de glutininas en las últimas semanas del embarazo.

En cuanto a la vía dei parto también existe controversia. Considerando que el parto espontáneo por las contracciones uterinas podría determinar un mayor paso de anticuerpos al feto, se recomendó con mucho entusiasmo la cesárea; con ella también se 
disminuía el traumatismo inevitable de todo parto por las vías naturales. Estadísticas comparativas demostraron luego que era mayor la mortalidad entre los niños obtenidos por medio de cesárea y existe actualmente la tendencia a dejar el parto por las vías naturales. En dos de nuestras pacientes en quienes por sus antecedentes se deseaba una conducta de seguridad se practicó la cesárea con resultados buenos.

En medios más propicios que el nuestro, los elementos para 'a investigación y tratamiento de la eritroblastosis están disponibles en cualquier momento. Esta diferencia de nuestro medio nos ha hecho usar el parto inducido artificialmente, prematuro $o$ a término en varias de nuestras pacientes, con antecedentes de hijos eritroblastósicos, con el fin de poder tener a mano para una hora determinada, todos los elementos y personal para la investigación y tratamiento de la enfermedad. Todos sabemos que los dadores $\mathrm{Rh}$ negativos no son numerosos y puede afirmarse aue los malos resultados de muchos casos se han derivado de la tardanza de algunas horas en la determinación del diagnóstico y la iniciación del tratamiento. No hemos tenido ningún muerto en los casos de parto provocado artificialmente.

Investigación y diagnóstico de la enfermedad.-Una vez nacido el niño y seccionado el cordón umbilical, sobre el extremo placentario de éste se punciona la vena umbilical y se obtienen 10 c c. de sangre la cual se distribuye en dos tubos, uno con antigoagulante y el otro sin él. El procedimiento de exprimir el cordón para obtener la sangre determina hemolisis o la coagula rápidamente, con lo cual la muestra resuita inapropiada. La muestra sin anticoagulante se destina a la investigación de bilirrubina (directa e indirecta), y aglutininas bivalentes y monovalentes (Coombs indirecto). La muestra con anticoagulante se usará para la prueba de grupo sanguíneo, $\mathrm{Rh}$, Coombs directo, cuadro hemático e investigación de eritroblastos. Debido a que con mucha frecuencia esta sangre resulta inadecuada por su fácil coagulabilidad, se recomienda hacer siempre una punción del talón con lanceta y una vez que se haya obtenido sangre, abocar sobre la gota un tubo que contiene una solución de oxalato de potasio al $0,5 \%$, los glóbulos así obtenidos sirven para practicar la prueba de Coombs directa y el Rh. A continuación se obtiene directamente de la gota y con la pipeta para hemograma, la muestra destinada al cuadro hemático. El muñón umbilical del niño se cubrirá con una gasa la cual se mantendrá embebida en solu- 
el embarazo, la conducta más juiciosa es esperar los datos del laboratorio. Si el niño manifiesta signos clínicos bastará con el dato del Coombs directo positivo de cuyo valor estamos convencidos. Si el niño aparenta ser normal en todo sentido, debe disponerse de toda la investigación ordenada. La bilirrubina indirecta por encima de 5 miligramos por ciento y la prueba de Coombs directa positiva son los datos que más ayudan en la definición de la conciucta. El número de glóbulos rojos no tiene en realidad el gran valor que antes se la atribuía pues nosotros mismos hemos presenciado eritroblastosis graves con cifras de 5 millones de glóbulos rojos. Sin embargo, la presencia de anemia es un dato adyuvante de gran valor si se excluyen otras causas de anemia (3). El número de eritroblastos tiene valor si se halla aumentando, pero de ninguna manera es dato excluyente cuando no se le encuentra. La cifra máxima que hemos encontrado es de 31.350 por $\mathrm{mm} 3$; pero comunmente solo encontramos entre 400 y 1.000 céiulas rojas nucleadas por $\mathrm{mm} 3$. Nos ha extrañado que estas cifras están dentro de las dadas para niños normales en el momento del nacimiento (4). No está por demás recordar que frecuentemente el laboratorio da el dato de eritroblastos en una cifra por cada 100 glóbulos blancos; como el número de estos es muy variable en 'a eritroblastosis, se obtiene una mejor idea haciendo la proporción para obtener el dato por $\mathrm{mm} 3$. En fin, la presencia de aglutininas (bivalentes o monovalentes) en la sangre del cordón coadyuva al diagnóstico.

Si el niño aparece sano y el laboratorio no suministra datos de certeza, el procedimiento a seguir debe ser el de la observación clínica frecuente y los exámnes seriados de cuadro hemático, Coombs directo y bilirrubina indirecta. La aparición de ictericia y espienomegalia en las prmeras 24 horas casi siempre se ve acompañada de su correspondiente comprobación por parte del laboratorio (disminución de la cifra de glóbulos rojos y aumento de la bilirrubina indirecta).

Hasta aquí nos hemos referido a los casos que podríamos llamar "modelos", es decir, clientela obstétrica bien conducida con investigación sistemática del factor $\mathrm{Rh}$ y control bien ilevado de las gestantes $\mathrm{Rh}$ negativas. Desafortunadamente entre nosotros todavía la mayor parte de los casos se refieren a la consulta por el recién nacido de tres o cuatro días que presentan una ictericia acentuada. Generalmente se trata de madres sin antecedentes de hijos eritroblastósicos, por lo menos en las ciudades cuan- 
do tal antecedente existe o se sospecha, la mayoria de los obstetras investigan el $\mathrm{Rh}$. En esos casos, una anamnesis más minuciosa con frecuencia permite hallar algún dato de interés; una transfusión incompatible después del penúltimo hijo (caso 12), jctericias leves en los hijos anteriores o un aborto tratado con legrado en el penúltimo embarazo (caso 9). Otras veces la anamnesis es completamente negativa. Pero si el cuadro clínico lo justifica, debe procederse rápidamente a la investigación de laboratorio y con el solo dato de madre $\mathrm{Rh}$ negativo, niño $\mathrm{Rh}$ positivo y Ccombs directo positivo, hacer la exanguinotransfusión lo más pronto posible para "ganarle la carrera" al ascenso de la biiirrubina pues es sabido que cifras superiores a 19 miligramos por ciento engloban el peligro inminente de la ictericia nuclear.

Generalmente en estos niños de tres o cuatro días el cuadro clínico es completo y permite hacer con relativa facilidad la disiinción con otras entidades; en orden de frecuencia están: ictericia acentuada con coluria e hipercolia, espienomegalia y hepatomegalia, edemas y estado general deficiente con gran indiferencia por la alimentación. Con alguna frecuencia, aún en esta situación no hay anemia, ni se encuentra cifra elevada de eritroblastos, pero casi constantemente la prueba de Coombs directa es positiva y la investigación en la madre es afirmativa. En dos de nuestros casos hemos hallado ya al niño con ia temible complicación de la ictericia nuclear: miembros superiores en hiper extensión con pronación y flexión de la mano, opistótonos, contracción del orbicuiar de los labios, estrabismo y nistagmus. Ante esta situación cabe el diagnóstico diferencial con las septicemias con meningitis que dan ictericia, signos neurológicos y hasta espienomegalia, siendo imprescindible el concurso del laboratorio para su diferenciación. Las hemorragias intracraneanas dan signos neurológicos parecidos e ictericia; ésta sin embargo aparece más tardíamente y no es tan acentuada; no hay esplenomegalia y las condiciones en que se verificó el parto permiten sospecharla. Pero como pueden coexistir las dos entidades, ante la duda, cabe practicar la investigación de laboratorio para eritroblastosis. En ausencia de Kernicterus, el diagnóstico diferencial debe dirigirse especialmente a la llamada ictericia fisiológica, (de aparición al tercer día y que no afecta el estado general del niño) sobre todo cuando se trata de prematuros y es acentuada, para lo cual es inćispensable la ayuda del laboratorio. En la ictericia por estrechez congénita de las vías biliares, el dato de Acolia tiene fundamental importancia. Sin embargo, como ya lo hicimos notar en un traba- 
jo recientemente publicado sobre este tema (5), en la eritroblastosis fetal, el gran acúmulo de pigmentos biliares producto de la destrucción hemática, puede ocasionar la obstrucción de las vías biilares por barro biliar y determinar la tríada sintomática ictericia, acolia y coluria; sin embargo la acolia es intermitente pero como este dato no es fácil de obtener, es lo mejor investigar siempre la eritroblastosis también en las ictericias que parezcan obstructivas. Una nueva entidad se ha ofrecido v́ltimamente al diagnóstico diferencial en casos de anemia acentuada del recién nacido: se trata de la anemia que sufre el feto por hemorragia intrapiacentaria, es decir, por ruptura de las vellosidades coriales con paso de la sangre del feto a los lagos sanguíneos de la placenta (3). En estos casos se encuentra anemia acentuada pero sin єsplenomegalia y la investigación serológica y hematológica para eritroblastosis es negativa. Este cuadro clínico requiere transfusión pero no exanguinotransfusión. En su publicación original Wiener describe un interesante caso de asociación de las dos entidades y se considera que la hemorragia "dei feto en la madre" favoreció la isoinmunización masiva de ésta. Nosotros también observamos un caso semejante en una primigestante Rh negativa. El recién nacido puede sufrir anemia aguda también en casos de inserción velamentosa del cordón (6) y es este un motivo más para ei examen minucioso de toda placenta. En fin, ante un caso de eritroblastosis clínica en que la investigación para $\mathrm{Rh}$ no la confirma, debe pensarse en los otros factores susceptibles de dar isoinmunización, lo cual será tema de la segunda parte de este trabajo.

Tratamiento.-En relación al tratamiento de la eritroblastosis fetal nos referimos solo a las conclusiones prácticas de 10 cue hasta ahora hemos podido realizar, dejando de lado recomendaciones que no sean practicables en nuestro medio. Consideramos la exanguinotransfusión como un procedimiento por medio del cual se proporcionan al niño glóbulos rojos no hemolizables mientras se extraen los suyos propios (hemolizábles) para mantenerlo en condiciones de salud mientras elimina espontáneamente los anticuerpos transmitidos por la madre. Es la opinión más aceptada que la aparición de Kernicterus está relacionada intimamente con el ascenso de la bilirrubina sérica, vale decir con la intensidad de la hemolisis. Por eso, aunque no desconocemos las opiniones en contra de esta teoría, somos partidarios de la exanguinotransfusión en todo caso de eritroblastosis, y de usar siempre que sea posible, sangre $\mathrm{Rh}$ negativa. 
Desechamos así la práctica de las pequeñas transfusiones repetidas por considerar que ellas solo tratan de corrgir la anemia, que no siempre existe y que no es el principal problema; permiten que siga la hemolisis de los glóbulos propios del niño con aumento peligroso de la bilirrubina sérica y aumentan el tenor de conglutinina (proteina X o complemento) (7), favoreciendo la aglutinación de los glóbulos rojos bloqueados. Por otra parte, de 7 casos que conocemos, propios y por referencias, tratados con pequeñas transfusiones, todos han muerto, algunos por Kernicterus. Uno solo sobrevivió con numerosísimas transfusiones que requirieron un período muy largo de hospitalización, mayores gastos y traumatismos para el niño.

La escogencia de la sangre se ha prestado recientemente entre nosotros a una interesante controversia entre pediatras de Barranquilla y Medellín (8). Si bien es cierto que se han reportado buenos resultados con el uso de sangre $\mathrm{Rh}$ positiva aún que sea por estar de acuerdo con las teorías más aceptadas, la sangre de elección es la Rh negativa. Porque no se trata de "lavar" al niño de anticuerpos y bilirrubina, cosa por demás imposible toda vez que estas substancias se encuentran en grandes concentraciones no solo en la sangre circulante sino en las células mismas y tejido intersticial de todo el organismo. Nosotros hemos visto cómo desjués de la exanguinotransfusión la bilirrubina apenas sí desciende unos pocos miligramos y lo mismo los anticuerpos. Tampoco se trata de reemplazar simplemente la sangre pobre' en glóbuios del niño por la normal del dador, porque no siempre existe anemia y además, no es esta la que mata al niño. Muy por el contrario, la exanguinotransfusión tiene por objeto, como ya lo hemos dicho, primordialmente suministrar al niño glóbulos rojos no hemolizables, extrayéndole los suyos propios hemolizables $\mathrm{y}$ esto solo se consigue usando sangre $\mathrm{Rh}$ negativa. Cuando al cabo de unos meses la sangre del dador se haya destruído, los glóbulos rojos $\mathrm{Rh}$ positivos del niño que ia han reemplazado ya no se destruirán porque habrá eliminado espontáneamente gran parte de los anticuerpos transmitidos por la madre. En cambio, si se usa sangre Rh positiva, lo único que se consigue es corregir la anemia, pero lógicamente la hemolisis aumentará y la bilirrubina alcanzará los niveles que se consideran peligrosos para el sistema nervioso. Ante la imposibilidad de encontrar un dador Rh negativo, pueden usarse los glóbulos rojos lavados de la madre, suspendidos en plasma humana; pero si las condicio- 
nes de ésta no permiten una extracción de sangre, la única solución de emergencia sería usar sangre $\mathrm{Rh}$ positiva.

Resultados.-De los 15 casos de eritroblastosis por isoinmunización anti $\mathrm{Rh}$, en 12 se ha verificado la exanguinotransfusión por la vía umbilical y en dos utilizando la vena safena y la arteria radial. En uno no se alcanzó a realizar el tratamiento (hidropesía fetal). No hemos tenido ninguna muerte durante el procedimiento. Las otras dos defunciones (casos 3 y 12) correspondieron la primera a un caso muy grave desde el nacimiento con insuficiencia cardíaca congestiva, estertores puimonares y anasarca; la segunda un caso en el que a pesar de haberse presentado ictericia desde las 12 horas del nacimiento, por una falsa seguridad dada por un examen previo según el cual la madre era Rh positiva, no se nos consultó sino al tercer día cuando el niño acusaba signos de kernicterus y estertores pulmonares. En uno cie los niños (caso 4) la exanguinotransfusión se hizo cuando ya se esbozaban signos de kernicterus (opistótonos y estrabismo); lo vimos por última vez a la edad de nueve meses y acusaba retardo neuropsíquico moderado. Igual cosa ocurre con nuestro primer caso, a quien ya en presencia de signos de kernicterus se hizo una exanguinotransfusión parcial que permitió su supervivencia pero a la edad de diez y siete meses acusa algún retardo neurológico. En dos casos más los signos de kernicterus se hicieron presentes después de la exanguinotransfusión: en uno de ellos (caso 2, hermano del caso 1), a al edad de siete meses se aprecia moderado retardo neuropsíquico; en cambio el otro (caso 7), a la edad de doce meses parece completamente normal. En el período inmediato a la exanguinotransfusión hemos tenido en 4 casos los problemas de obstrucción biliar de que hemos hablado, por espesamiento de la bilis consecutivo a la gran hemolisis. Hemos usado por dos o tres días la solución de sulfato de magnesio al $33 \%$ por vía oral como fluidificante de la bilis, con buenos resultados. En casos rebeldes podría usarse el dehidrocolato de sodio por vía venosa que es el procedimiento de elección en estos casos (5), debido al efecto depresor que tiene el sulfato de magnesio cuando se usa por varios días. Se presentó un caso de edema genreaslizado (caso 6), el cual consideramos en principio como fracaso del tratamiento con agravación de la enfermedad pero que cedió en 24 horas con la administración de dosis altas de cloruro de calcio, por lo cual consideramos que pudiera tener relación más bien con el exceso de ión sodio suministrado en la exanguinotransfusión. 